\title{
Liquid-Vapor Density Profile of Helium: An X-Ray Study
}

\section{Citation}

Lurio, L. B., T. A. Rabedeau, Peter S. Pershan, Isaac F. Silvera, M. Deutsch, S. D. Kosowsky, and B. M. Ocko. 1992. Liquid-vapor density profile of helium: An x-ray study. Physical Review Letters 68(17): 2628-2631.

\section{Published Version}

doi:10.1103/PhysRevLett.68.2628

\section{Permanent link}

http://nrs.harvard.edu/urn-3:HUL.InstRepos:10357544

\section{Terms of Use}

This article was downloaded from Harvard University's DASH repository, and is made available under the terms and conditions applicable to Other Posted Material, as set forth at http:// nrs.harvard.edu/urn-3:HUL.InstRepos:dash.current.terms-of-use\#LAA

\section{Share Your Story}

The Harvard community has made this article openly available.

Please share how this access benefits you. Submit a story.

Accessibility 


\title{
Liquid-Vapor Density Profile of Helium: An X-Ray Study
}

\author{
L. B. Lurio, ${ }^{(1)}$ T. A. Rabedeau, ${ }^{(2)}$ P. S. Pershan, ${ }^{(1),(2)}$ Isaac F. Silvera, ${ }^{(1)}$ M. Deutsch, ${ }^{(2),(a)}$ \\ S. D. Kosowsky, ${ }^{(1)}$ and B. M. Ocko ${ }^{(3)}$ \\ (1)Department of Physics, Harvard University, Cambridge, Massachusetts 02138 \\ ${ }^{(2)}$ Division of Applied Sciences, Harvard University, Cambridge, Massachusetts 02138 \\ (3) Physics Department, Brookhaven National Laboratory, Upton, New York 11973-5000
}

(Received 22 July 1991; revised manuscript received 25 March 1992)

\begin{abstract}
The average liquid-vapor density profiles $\langle\rho(z)\rangle$ of thick ${ }^{4} \mathrm{He}$ films adsorbed onto a silicon substrate were measured using $x$-ray reflectivity. The results are well represented by a $90 \%-10 \%$ interfacial width of $9.2 \pm 1 \AA$ at $1.13 \mathrm{~K}$ which extrapolates to a $T=0 \mathrm{~K}, 90 \%-10 \%$ interfacial width of $7.6 \pm 1 \AA$. The sensitivity of the measurement to the width, shape, and asymmetry of the density profile is discussed.
\end{abstract}

PACS numbers: $67.70 .+\mathrm{n}, 61.25 . \mathrm{Bi}, 68.15 .+\mathrm{e}$

Structural characterization of the ${ }^{4} \mathrm{He}$ liquid-vapor interface is essential to the understanding of basic features of quantum fluids [1], as well as to the understanding of layers of ${ }^{3} \mathrm{He}$ on ${ }^{4} \mathrm{He}$ [2], electrons on liquid ${ }^{4} \mathrm{He}[3]$, and spin-polarized atomic hydrogen with ${ }^{4} \mathrm{He}$-coated confining walls [4]. While there have been many theoretical predictions for the ${ }^{4} \mathrm{He}$ liquid-vapor profile [5-11], the experimental determination of this profile remains incomplete. Atomic scattering experiments, for example, are sensitive to the vapor-side tail of the profile but not to its inner, higher-density region [12]. Ellipsometry is sensitive to the width, but not the shape of the profile [13]. By contrast, $x$-ray specular reflectivity provides angstromlevel resolution of the interfacial electron density profile [14]. In this Letter we report such measurements on the free surface of superfluid ${ }^{4} \mathrm{He}$. We have not only determined the width, but also the surface profile of helium films adsorbed onto a silicon substrate, over a range of temperatures and film thicknesses.

For $\mathrm{x}$ rays of wavelength $\lambda \sim 1 \AA$ the specular reflectivity from an arbitrary interface between vapor and a bulk material of electron density $\rho_{\infty}$ can be calculated using the first Born approximation whenever the grazing angle of incidence $\theta \gtrsim 5 \theta_{c}$. Here $\theta_{c} \equiv\left[\rho_{\infty} r_{e} \lambda^{2} / \pi\right]^{1 / 2}$ is the critical angle for total external reflection, with $r_{e}$ the classical electron radius, $\lambda$ the wavelength of the incident radiation, and $\rho_{\infty}$ the electron density of the bulk material. Defining $q \equiv 4 \pi \sin (\theta) / \lambda$ and $R_{F}(q)$ as the Fresnel reflectivity from a sharp flat interface between the vacu- um and the same bulk material, the reflectivity is given by the relation $R(q)=R_{F}(q)|S(q)|^{2}$. Here the structure factor of the interface is given by

$$
S(q)=\frac{1}{\rho_{\infty}} \int_{-\infty}^{\infty}[d\langle\rho(z)\rangle / d z] \exp (i q z) d z,
$$

where $\langle\rho(z)\rangle$ is the electron density at position $z$ along the surface normal averaged over the $x-y$ coherence area of the incident $x$-ray beam in the plane of the surface $[15,16]$. For $q \lesssim 5 q_{c}$, the above expression for $R(q)$ is not accurate, and a full dynamical formalism must be employed [17].

Since the reflectivity from a semi-infinite slab of helium scales as $\rho_{\mathrm{He}}^{2}$, and $\rho_{\mathrm{He}}$ is small, direct measurement of $R(q)$ is technically difficult (e.g., $R_{\mathrm{He}} / R_{\text {water }} \approx 10^{-2}$ ). We employed therefore a geometry in which interference between $\mathrm{x}$ rays reflected from the front and back surfaces of a film of ${ }^{4} \mathrm{He}$ adsorbed onto an atomically flat silicon wafer was measured. Theoretical models of correlations between the surface structures of a substrate and an absorbed film indicate that for thick films the influence of the substrate on the local properties of the free surface are negligible [18] and this is supported by roomtemperature studies of organic liquid films [19]. Hence, we believe that the surface of a thick ${ }^{4} \mathrm{He}$ film should accurately resemble that of bulk ${ }^{4} \mathrm{He}$. If the film is sufficiently thick that the two interfaces can be regarded as independent, $R(q)$ can be expressed in terms of their structure factors, $S_{\mathrm{Si}}(q)$ and $S_{\mathrm{He}}(q)$ :

$$
\begin{aligned}
|S(q)|^{2} & =\left|A S_{\mathrm{Si}}(q)+B S_{\mathrm{He}}(q) e^{i q d_{\mathrm{film}}}\right|^{2} \\
& =A^{2}\left|S_{\mathrm{Si}}(q)\right|^{2}+B^{2}\left|S_{\mathrm{He}}(q)\right|^{2}+2 A B \cos \left[q d_{\mathrm{film}}+\phi_{\mathrm{He}}(q)-\phi_{\mathrm{Si}}(q)\right]\left|S_{\mathrm{Si}}(q)\right|\left|S_{\mathrm{He}}(q)\right| .
\end{aligned}
$$

Here $A=1-\rho_{\mathrm{He}} / \rho_{\mathrm{Si}}, B=\rho_{\mathrm{He}} / \rho_{\mathrm{Si}}, d_{\mathrm{film}}$ is the thickness of the film, $\phi_{\mathrm{Si}}(q)$ and $\phi_{\mathrm{He}}(q)$ are the phases of the complex $S_{\mathrm{Si}}(q)$ and $S_{\mathrm{He}}(q)$, and $\rho_{\mathrm{Si}}$ and $\rho_{\mathrm{He}}$ are the mean electron densities of bulk silicon and liquid helium. The first two terms represent the reflection of $\mathrm{x}$ rays from the Si-liquid interface and the liquid-vapor interface, respectively. Since $B^{2} \approx(0.06)^{2}$ the second term is negligible compared to the first. By contrast, the third term, representing interference between reflections from the top and bot- tom interfaces of the film, is $\sim 13 \%$ of the first term with an oscillating amplitude proportional to $\left|S_{\mathrm{He}}(q)\right|$. Hence for the interference technique, there is an increase by a factor of $1 / 0.06 \approx 17$ in the part of the signal proportional to $S_{\mathrm{He}}(q)$ compared to reflectivity from a surface of bulk ${ }^{4} \mathrm{He}$. In principle, the interference technique can also be sensitive to the relative phase difference between the two structure factors, $\phi_{\mathrm{He}}(q)-\phi_{\mathrm{Si}}(q)$. This would 
enable the determination of the asymmetry of the density profile of the helium interface when the asymmetry for the silicon interface is known. This point will be discussed below.

Measurements were performed at the National Synchrotron Light Source on beam line X-22B, using a pumped ${ }^{4} \mathrm{He}$ cryostat mounted on a horizontal two-circle diffractometer. A polished, hydrogen-passivated silicon substrate with the normal to the $\mathrm{Si}(111)$ surface horizontal $[20,21]$ was placed inside a sealed cell within the cryostat. Measurements were performed between 1.1 and 3.0 $\mathrm{K}$ both on saturated films, where for $T \leq 4 \mathrm{~K}$ a pool of bulk liquid condensed at the bottom of the cell, and on undersaturated films, where for a fixed dose of ${ }^{4} \mathrm{He}$ the thickness of the film depended on the temperature of the cell. In addition, measurements were made at $20 \mathrm{~K}$ on the dry silicon substrate. In the case of saturated films, the theoretical $d_{\text {film }}$ varies with height $h$ above the condensed ${ }^{4} \mathrm{He}$ pool as $\gamma\left(d_{\text {film }}\right) / d_{\text {film }}^{3}=-\Delta \mu=m_{\mathrm{He}} g h$ [22]. Here $\Delta \mu$ is the change in chemical potential with respect to the bulk, $m_{\mathrm{He}}$ is the mass of a ${ }^{4} \mathrm{He}$ atom, $g$ is the acceleration of gravity, and $\gamma$ is the strength of the van der Waals interaction. From the period of interference fringes that were observed for saturated films, e.g., similar to those shown in Fig. 1, the film thickness was measured to have a temperature-independent value of $215 \pm 1$ $\AA$ at $h=2.5 \pm 0.2 \mathrm{~cm}$, which yields $\gamma(215 \AA)=1192$ $\pm 95 \mathrm{KA}^{3}$ as compared to the theoretical prediction $1023 \mathrm{~K} \AA^{3}$ of Cheng and Cole [22]. The discrepancy between the experimental and theoretical numbers is reduced when phonon-induced effects, as described by Cole [23], are included. For an undersaturated film the thickness is determined by the condition $\gamma\left(d_{\text {film }}\right) / d_{\text {film }}^{3}=-\Delta \mu$ $=k_{B} T \ln \left(P_{\text {sal }} / P\right)$, where $P_{\text {sat }}$ is the saturation vapor pressure; however, as the amount of ${ }^{4} \mathrm{He}$ in the cell was fixed in the experiments described here $P$, and hence $d_{\text {film }}$, depended only on the cell temperature.

Extraction of the structural information from measurements using the present geometry, in which the ${ }^{4} \mathrm{He}$ surface structural information is carried by the envelope of the interference oscillations, can be complicated by effects such as partial or nonuniform coverage of the substrate or any uncontrolled loss of phase coherence between waves reflected from the two interfaces. These effects can be assessed from data at small $q$, since for $q \rightarrow 0, S(q) \cong 1$ [e.g., Eq. (1)], independent of smalllength-scale details of the interfacial profiles. Although for saturated films the amplitude of the interference fringes was systematically lower than the theoretical values, for undersaturated films, the fringe amplitude agreed perfectly with the results predicted from a dynamical treatment of the reflectivity [17]. We suspect that the source of the missing amplitude for saturated films can be attributed to the coupling of external vibrations to macroscopic motions of the condensed puddle of bulk ${ }^{4} \mathrm{He}$. Above $T_{\lambda}$ the thickness of the normal-fluid ${ }^{4} \mathrm{He}$ film was sensitive to incident $x$-ray flux in excess of $10^{8}$ photons $/ \mathrm{cm}^{2} \mathrm{sec}\left(\sim 0.1 \mu \mathrm{W} / \mathrm{cm}^{2}\right)$ and for the maximum incident flux of $5 \times 10^{11}$ photons $/ \mathrm{cm}^{2} \mathrm{sec}$ the film thickness decreased from 215 to $50 \AA$, i.e., comparable to that due to a thermal gradient $\Delta T \sim 0.01 \mathrm{~K}$. By contrast, for superfluid films, the thickness was independent of incident flux. In this Letter we focus on thick undersaturated superfluid films.

Figure 1 shows the measured ratios $R(q) / R_{F}^{S i}(q)$ for a silicon substrate coated with undersaturated ${ }^{4} \mathrm{He}$ films at four different temperatures, corresponding to four different film thicknesses. In addition the bare silicon substrate reflectivity at $20 \mathrm{~K}$ is shown for comparison. The data were analyzed by calculating the theoretical reflectivity of a model $d\langle\rho\rangle / d z$, and optimizing the model parameters to obtain a best fit between the calculated and measured reflectivities.

To facilitate construction of a model $d\langle\rho\rangle / d z$ for the substrate-film interface, independent scanning tunneling microscopy (STM) and x-ray photoelectron spectroscopy (XPS) analysis of the bare-substrate-vapor interface was performed [16]. This analysis indicates that the bare substrate surface consisted of flat $\sim 300-\AA$ terraces covered by a partial monolayer of hydrocarbon contamination. These features of the bare substrate were incorporated into a model used to represent the scattering from the bare substrate at $20 \mathrm{~K}$ (Fig. 1). In the presence of the ${ }^{4} \mathrm{He}$ film the substrate-film interface was modified to include a thin layer of solid ${ }^{4} \mathrm{He}$. The best fits to the reflectivity data required a $5-6-\AA$ solid film of density $1.8 \rho_{\mathrm{He}}$, which roughly corresponds to the two solid layers found in third-sound studies of ${ }^{4} \mathrm{He}$ films [24].

Although the detailed study of dry silicon lends confidence to our model for the wet substrate surface, to a large extent, extraction of the ${ }^{4} \mathrm{He} /$ vapor interface is independent of the model used for the $\mathrm{Si} /{ }^{4} \mathrm{He}$ interface. For a thick helium film the reflectivity oscillates about

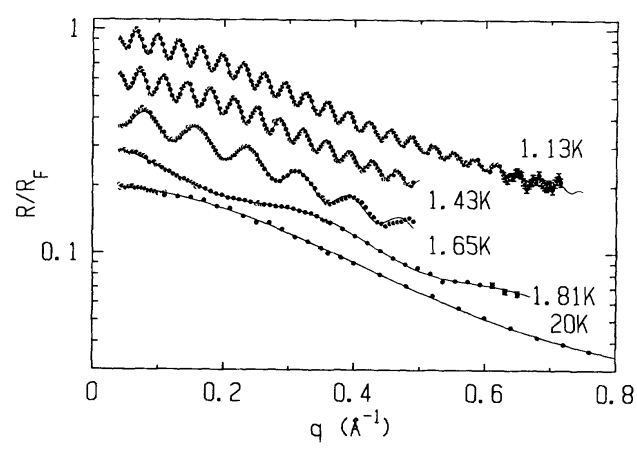

FIG. 1. $R(q) / R_{F}^{S i}(q)$ for a silicon substrate coated with several different undersaturated ${ }^{4} \mathrm{He}$ films: Top to bottom; $T=1.13 \mathrm{~K}, d_{\text {film }}=192 \AA ; T=1.43 \mathrm{~K}, d_{\text {film }}=175 \AA ; T=1.65 \mathrm{~K}$, $d_{\text {film }}=80 \AA ; T=1.81 \mathrm{~K}, d_{\text {film }}=18 \AA ; T=20 \mathrm{~K}$ without a film. Data are shown as circles; lines represent fits to data using the asech model convoluted with thermal capillary waves as described in the text. Successive data sets are multiplied by 0.67 for clarity. 
the value $A^{2}\left|S_{\mathrm{Si}}(q)\right|^{2}$ and the product $2 A B\left|S_{\mathrm{Si}}(q)\right|$ $\times\left|S_{\mathrm{He}}(q)\right|$ can be independently extracted from the envelope of the oscillations in $R(q)$. Similarly, the phase difference $\phi_{\mathrm{He}}(q)-\phi_{\mathrm{Si}}(q)$ is obtained directly from the phase of the oscillations in $R(q)$. It is straightforward to demonstrate that if $d\langle\rho(z)\rangle / d z$ were locally symmetric about positions $z_{\mathrm{Si}}$ and $z_{\mathrm{He}}$ that defined the center of the $\mathrm{Si} /{ }^{4} \mathrm{He}$ and ${ }^{4} \mathrm{He} /$ vapor interfaces, then $\phi_{\mathrm{Si}}(q)=\phi_{\mathrm{He}}(q)$ $=0$. Thus if the interfaces were symmetric, $S_{\mathrm{He}}(q)$ would be directly obtained from the data, regardless of whether or not the model for the $\mathrm{Si} /{ }^{4} \mathrm{He}$ interface was valid. As a practical matter, since the first derivative can be subsumed within the definition of the thickness and the data do not extend to large enough $q$ to consider higher derivatives, the only feature of the present data that yields physical information on the asymmetry of either interface is $d^{2}\left[\phi_{\mathrm{Si}}(q)-\phi_{\mathrm{He}}(q)\right] / d q^{2}$. Since $\left|S_{\mathrm{He}}(q)\right|$ is all that would have been obtained from specular reflectivity measurement of the bulk ${ }^{4} \mathrm{He} /$ vapor interface, no information obtainable from that method is lost in the interference measurement. In contrast, the factor of $\sim 17$ gain in amplitude using the interference method yields a concomitant improvement in the measurement of the width of the ${ }^{4} \mathrm{He} /$ vapor interface. Although extraction of $\phi_{\mathrm{He}}(q)$ from the phase difference $\phi_{\mathrm{Si}}(q)-\phi_{\mathrm{He}}(q)$ depends on details of the model for the silicon-helium interface, the use of both STM and XPS minimizes this source of uncertainty.

The measurements reported here were made above $1 \mathrm{~K}$ and the profiles are broadened by thermally excited capillary waves or ripplons. Cole [25] has calculated this broadening, using the ripplon model of Atkins [26]. We apply this calculation to the present experiment. Since the total capillary-wave roughness is an incoherent superposition of many modes, the net profile is a convolution of the $0-\mathrm{K}$ profile with a Gaussian whose second moment is calculated from Cole's theory:

$$
\frac{d\left\langle\rho\left(z, d_{\text {film }}, T\right)\right\rangle_{\mathrm{He}}}{d z}=\frac{1}{\left(2 \pi \sigma_{\text {cap }}^{2}\right)^{1 / 2}} \int_{-\infty}^{\infty} \exp \left(-z^{\prime 2} / 2 \sigma_{\text {cap }}^{2}\right) \frac{d\left\langle\rho\left(z^{\prime}-z\right)\right\rangle_{0}}{d z} d z^{\prime} .
$$

Here $\sigma_{\text {cap }}^{2}\left(d_{\text {film }}, T\right)$ is the second moment of the capillarywave roughness at temperature $T$ and film thickness $d_{\text {film, }}$, and averaged over the coherence area of the $x$-ray beam, while $d\langle\rho\rangle_{0} / d z$ is the $0-\mathrm{K}$ density profile. Theoretical values of $\sigma_{\text {cap }}$ are listed in Table I for relevant values of $d_{\text {film }}$ and $T$. This procedure is advantageous as the $0-\mathrm{K}$ profile determined from data collected for films of different temperature and thickness can be compared for consistency and to theoretical predictions for the $0-K$ profile.

The solid lines in Fig. 1 represent best fits for model interfacial profiles with $\sigma_{\text {cap }}^{2}$ fixed at the calculated values shown in Table I. Several different ${ }^{4} \mathrm{He} /$ vapor functional forms were tested; however, the only one that gave satisfactory fits was an asymmetric hyperbolic secant (asech) model:

$$
\begin{aligned}
& \frac{d\langle\rho\rangle_{0}}{d z}=\frac{C}{\exp \left(a z / \sigma_{0}\right)+\exp \left(-b z / \sigma_{0}\right)}, \\
& a=1+\exp (-\eta), \quad b=1+\exp (\eta),
\end{aligned}
$$

where $\sigma_{0}$ is a measure of the interfacial width, $\eta$ represents the asymmetry of the interface (e.g., $\eta=0$ yields a symmetric interface), and $C$ is fixed such that $\int(d\langle\rho\rangle / d z) d z=\rho_{\mathrm{He}}$. Although the same model fits all four data sets with comparable values of $\chi^{2} \cong 0.9$, only the $T=1.13 \mathrm{~K}$ data extend to sufficiently large $q$ to obtain a good measure of $d^{2}\left[\phi_{\mathrm{Si}}(q)-\phi_{\mathrm{He}}(q)\right] / d q^{2}$. The solid lines shown in Fig. 1 correspond to fits using the values of $\sigma_{0}$ listed in the table and the value of $\eta$ obtained from the 1.13-K data [27]. While all the values for $\sigma_{0}$ obtained from the different films were consistent with each other, the slightly larger roughness of the thinnest film measured at $1.8 \mathrm{~K}$ is to be expected, since it is sufficiently thin to be affected by the roughness of the un-

\section{derlying substrate [16].}

The $0-\mathrm{K}$ profiles were obtained by taking the best fit to the measured profiles and then setting the thermal capillary-wave roughness $\sigma_{\text {cap }}\left(d_{\text {film }}, T\right)$ to zero. The heavy solid line in Fig. 2 is obtained by integrating $d\langle\rho\rangle_{0} / d z$ [Eq. (4)], using the values of $\sigma_{0}$ and $\eta$ for 1.13 $\mathrm{K}$, to obtain the best-fit $0-\mathrm{K}$ profile. The light solid line and the dashed line represent the most asymmetric ( $\eta$ $\rightarrow-\infty)$ and symmetric $(\eta=0)$ profiles consistent with our measurement of the $1.13-\mathrm{K}$ data set. The $0-\mathrm{K}$ profile has a $90 \%-10 \%$ width of $7.6 \AA$. The theoretical prediction of Stringari and Treiner [5], as shown by the open squares, is in excellent agreement with our measured profile. There have been a large number of other theoretical calculations for the $0-\mathrm{K}$ density profile and several of these are shown in Fig. 2(c).

Osborne [13] has recently reported an ellipsometric determination of the surface width of bulk helium of $9.36 \pm 1.5 \AA$ over the temperature range $1.4-2.3 \mathrm{~K}$. This gives excellent agreement with our measured width (in-

TABLE I. Results of fits to data at several different temperatures and film thicknesses. Shown are the values of the parameters for the asymmetric hyperbolic secant fit with $\eta=-1.1$, convoluted with capillary-wave roughness. The small overall variation in the theoretical values of $\sigma_{\text {cap }}$ results from the compensating effects of increasing temperature and decreasing film thickness.

\begin{tabular}{cccr}
\hline$T(\mathrm{~K})$ & $\sigma_{\text {cap }}(\AA)$ & $\sigma_{0}(\AA)$ & $d_{\text {film }}(\AA)$ \\
\hline 1.13 & 2.03 & $1.97 \pm 0.22$ & $192 \pm 1$ \\
1.43 & 2.30 & $1.84 \pm 0.17$ & $175 \pm 2$ \\
1.65 & 2.16 & $1.63 \pm 0.23$ & $80 \pm 2$ \\
1.81 & 1.40 & $2.01 \pm 0.22$ & $18 \pm 3$ \\
\hline \hline
\end{tabular}




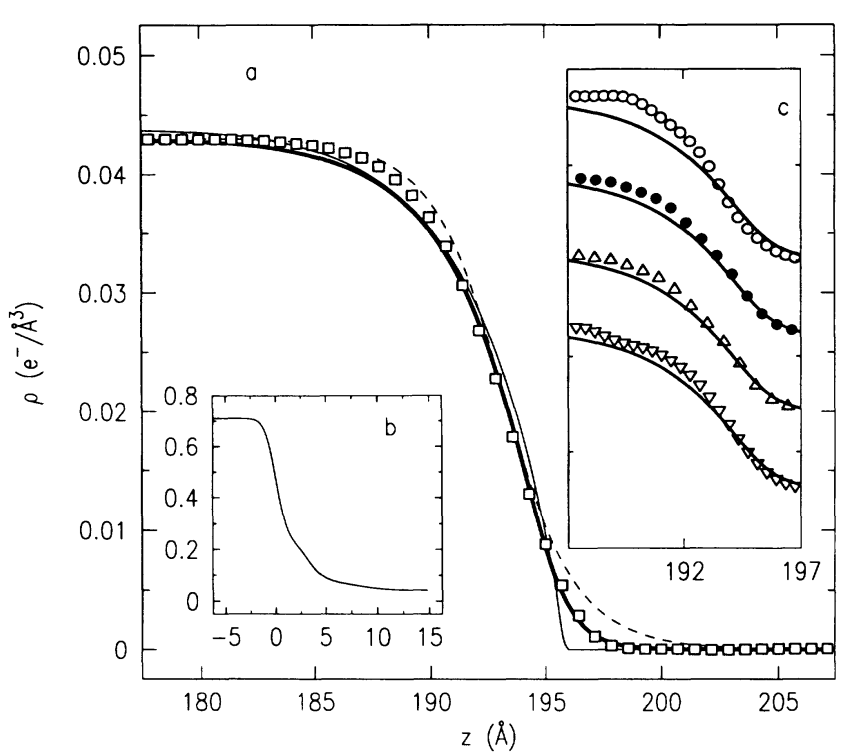

FIG. 2. (a) Density profile at the ${ }^{4} \mathrm{He} /$ vapor interface. The heavy solid line is the best-fit model extrapolated to $0 \mathrm{~K}$. The light solid line corresponds to largest asymmetry consistent with the data $(\eta \rightarrow-\infty)$. The dashed line corresponds to the smallest asymmetry $(\eta=0)$. Squares are the theoretical profile of Stringari and Treiner [5]. (b) Density profile at the $\mathrm{Si} /{ }^{4} \mathrm{He}$ interface. (c) Comparison of various theoretical profiles with our best-fit model (line). (O) Valles and Schmidt [9]; (๑) Pieper and Wiringa [8]; $(\Delta)$ Krishna and Whaley [11]; $(\nabla)$ Epstein and Krotscheck [6].

cluding thermal broadening) at $1.13 \mathrm{~K}$ of $9.2 \pm 1 \AA$. Atomic scattering measurements by Nayak, Edwards, and Masuhara, however, indicate a density profile with a $90 \%-10 \%$ width of $4 \AA$ at $T=0.02 \mathrm{~K}$ [12]. This width is significantly smaller than our $T=0 \mathrm{~K}$ extrapolation. Note, however, that atomic scattering measurements are most sensitive to the vapor side tail of the density profile. Comparing just the vapor side of the interface our best-fit profile gave a $50 \%-10 \%$ width of $2.6 \AA$, due to the asymmetry of the interface, in reasonable agreement with the width measured by Nayak, Edwards, and Masuhara.

We thank Ian Tidswell, John Mester, and John Brisson for helpful discussions. We also thank Hans Biebuyck for assisting with the $x$-ray photoelectron spectroscopy, and Ing-Shouh Hwang for assisting with the scanning tunneling microscopy measurement. This research was supported by Grants No. NSF-DMR-89-20490 and No. NSFDMR-88-12855. NSLS, Brookhaven National Laboratory, is supported by DOE Contract No. DE-AC02$76 \mathrm{CH} 00016$. (a) Permanent address: Physics Department, Bar Ilan University, Ramat Gan 52100, Israel.

[1] D. O. Edwards, Physica (Amsterdam) 109\& 110B, 1531 (1982).

[2] M. Himbert and J. Dupont-Roc, J. Low Temp. Phys. 76, 435 (1989).

[3] M. Cole, Rev. Mod. Phys. 46, 451 (1974).

[4] I. F. Silvera and J. T. M. Walraven, Prog. Low Temp. Phys. 10, 139 (1986).

[5] S. Stringari and J. Treiner, Phys. Rev. B 36, 8369 (1987).

[6] J. L. Epstein and E. Krotscheck, Phys. Rev. B 37, 1666 (1988).

[7] E. Krotscheck, S. Stringari, and J. Treiner, Phys. Rev. B 35, 4754 (1987).

[8] S. Pieper and R. Wiringa, Phys. Rev. B 32, 3341 (1985).

[9] J. Valles and K. Schmidt, Phys. Rev. B 38, 2879 (1988).

[10] W. Brouwer and R. Pathria, Phys. Rev. 163, 200 (1967).

[11] M. V. R. Krishna and K. B. Whaley, J. Chem. Phys. 93, 6738 (1990).

[12] V. U. Nayak, D. O. Edwards, and N. Masuhara, Phys. Rev. Lett. 50, 990 (1983).

[13] D. V. Osborne, J. Phys. Condens. Matter 1, 289 (1989).

[14] I. M. Tidswell, B. M. Ocko, P. S. Pershan, S. R. Wasserman, G. M. Whitesides, and J. D. Axe, Phys. Rev. B 41, 1111 (1990).

[15] A general reference supporting this for solids and liquids is A. Braslau, P. S. Pershan, G. Swislow, B. M. Ocko, and J. Als-Nielsen, Phys. Rev. A 38, 2457 (1988).

[16] L. B. Lurio, T. A. Rabedeau, P. S. Pershan, I. F. Silvera, M. Deutsch, S. D. Kosowsky, and B. M. Ocko (to be published).

[17] L. G. Parrat, Phys. Rev. 95, 359 (1954).

[18] D. Andelman, J. F. Joanny, and M. O. Robbins, Europhys. Lett. 7, 731 (1988).

[19] I. M. Tidswell, T. A. Rabedeau, P. S. Pershan, and S. D. Kosowsky, Phys. Rev. Lett. 66, 2108 (1991).

[20] G. S. Higashi, Y. J. Chabal, G. W. Trucks, and K. Raghavachari, Appl. Phys. Lett. 56, 656 (1990).

[21] On the basis of room-temperature $x$-ray reflectivity studies of different solids, hydrogen-passivated $\mathrm{Si}(111)$ was selected as the most homogeneous and macroscopically flat solid surface, with the sharpest interfacial profile. As a result the uncertainties in the phase of $S_{\mathrm{Si}}(q)$ are less for this surface than for any other that we examined.

[22] E. Cheng and M. Cole, Phys. Rev. B 38, 987 (1988).

[23] M. Cole (private communication).

[24] D. T. Smith and R. B. Hallock, Phys. Rev. B 34, 226 (1986).

[25] M. W. Cole, Phys. Rev. A 1, 1838 (1970).

[26] K. R. Atkins, Can. J. Phys. 31, 1165 (1953).

[27] The best-fit value of $\eta$ was -1.1 with a $2 \sigma$ confidence limit upper bound of 0 . No lower bound was found for $\eta$ at the $2 \sigma$ level. 